An anomalous patch of high temperature, associated with a cyclonic circulation resembling the terrestrial "heat low," was discovered and found to be located over a dark surface marking which appears seasonally in the Deucalion region.

Lowell Observatory, Flagstaff, Ariz.

\section{Hiltner, W. A. Photoelectric He II light curve of CQ Cephei.}

A photoelectric light curve of the $\mathrm{He}$ II 4686 emission line in the Wolf-Rayet spectroscopic binary CQ Cephei has been obtained at the McDonald Observatory. The curve shows two maxima and two minima. The emission is strongest at either conjunction and weakest at either elongation. This observed behavior of the emission is not understood but investigations of this character in this and other Wolf-Rayet stars may contribute toward a better understanding of Wolf-Rayet stars in general.

Yerkes Observatory, Williams Bay, Wis., and McDonald Observatory, Fort Davis, Texas.

Irwin, John B. Four-color photoelectric photometry of the globular clusters.

This is a preliminary report of photoelectric observations of the integrated colors of 73 globular clusters. The observations were made at the Cassegrain focus of the 82 -inch McDonald reflector and were made in four colors over a wave length range of approximately $2500 \mathrm{~A}$, using a IP2 I multiplier phototube. Four diaphragms were used whose diameters were $140^{\prime \prime}$, I06", $70^{\prime \prime}$ and $47^{\prime \prime}$. In general, two observations of each object were made; the internal agreement indicates a probable error of a single observation for clusters brighter than eleventh magnitude to be \pm o.oI I mag. for the B-Y, \pm 0.020 mag. for the $\mathrm{U}-\mathrm{Y}$, and \pm o.oro mag. for the $\mathrm{R}-\mathrm{Y}$ color indices. For the fainter clusters the corresponding errors are $\pm 0.012, \pm 0.048$ and $\pm 0.02 \mathrm{I}$ mag. The accuracy of the colors is such that good individual values of the color excess and hence good values of the total absorption should be obtainable, giving reliable distances for those clusters whose cluster-type variables have been obtained by accurate photometry. A plot of the $\mathrm{R}-\mathrm{Y}$ against the $\mathrm{U}-\mathrm{B}$ colors shows an increased scatter with reddening, a scatter greater than might be expected from the errors involved. The clusters of spectral type A show excellent agreement with the other clusters on this plot.
Further observations will be made on additional clusters; the tie-in with the North Polar Sequence and with the Stebbins-Whitford sixcolor photometry will be strengthened. These observations have been made possible through the cooperative agreement between Indiana University and the University of Chicago.

Goethe Link Observatory, Indiana University,

Keenan, Philip C. Bands in the photographic infra-red spectra of S-type stars.

The stars of type $\mathrm{S}$ are characterized by bands of zirconium oxide in the blue and yellow parts of their spectra. $\mathrm{ZrO}$, however, has only weak bands between 7500 and $8800 \mathrm{~A}$, and these do not contribute appreciably to S-type spectra in that region.

The cooler S-type stars show heavy band absorption in the near infra-red, the strongest bands being the known sequences near 7400 and $7900 \mathrm{~A}$. Grating spectrograms of $\mathrm{R} \mathrm{Cyg}$ (S3e) taken on Eastman I-N plates during August, I949, show the vibrational structure of these sequences and confirm their identification as bands of $\mathrm{LaO}$.

In addition, a number of new bands, degraded to the red, are present. The strongest of these have apparent heads at 8464, 86ro and $8820 \mathrm{~A}$. It may be expected that they are due to compounds, possibly oxides, of elements in the fourth or fifth periods of the periodic table.

Perkins Observatory, Delaware, Ohio.

Menzel, Donald H. and Hari K. Sen. Operational solution of radiative transfer in absorption lines.

The operational method previously described ${ }^{1}$ has been applied to solve the equation of the formation of absorption lines in the MilneEddington model of a solar or stellar atmosphere. The equation of transfer in the standard case reduces to the form

$$
\begin{aligned}
\mu \frac{\partial}{\partial \tau} I_{\nu}(\tau, \mu)= & I_{\nu}(\tau, \mu)-\frac{1}{2}(\mathbf{I}-\lambda) \\
& \times \int_{-1}^{+1} I_{\nu}(\tau, \mu) d \mu-\lambda(a+b \tau)
\end{aligned}
$$

where $\tau$ is the optical depth in the total (line and continuous) absorption, $\lambda$ is the ratio of the continuous to the total absorption coefficient, and $a$ and $b$ are the first two coefficients of the 